\title{
El clima, la ecología y el caos desde la perspectiva de la teoría general de sistemas
}

\author{
N. Sánchez-Santillán y R. Garduño-López \\ Departamento El Hombre y su Ambiente, UAM-Xochimilco y \\ Centro de Ciencias de la Atmósfera, UNAM, México \\ E-mails:santilla@correo.xoc.uam.mxy rene@atmosfera.unam.mx
}

(Recibido: noviembre de 2005; aceptado: diciembre de 2006)

\begin{abstract}
Resumen
La ciencia clásica determinista afronta dificultades para abordar y explicar fenómenos complejos, como son aquellos que conciernen al estudio del clima, la ecología o las sociedades humanas. Un nuevo paradigma científico, como la visión holística, ha ido llenando gradualmente los huecos dejados por el viejo enfoque mecanicista y ha incorporado, entre otras, además del mecanicismo de Newton, la teoría de la información, la cibernética, la teoría del caos y la teoría general de sistemas. Se analizan éstas dos últimas teorías con una somera aproximación a problemas de climatología y ecología.
\end{abstract}

Descriptores: Sistemas, caos, clima, ecología, modelos.

\begin{abstract}
Classic determin is tic science con fronts difficul ties to ap proach andex plain com plex phe nom ena, like those con cern ing the study of cli mate, ecol ogy or hu man so ciet ies. A new sci en tific par a digm, the ho lis tic vi sion, has been grad $u$ ally fill ing the gaps left by the old mechanicist ap proach, and along with New ton's mechanicism, has in cor po rated In for ma tion the ory, cybernetics, chaos theory and, general systems theory, among others. These last two theories arean alyzed with a su per ficial ap proach to cli ma tologyand ecology is sues.
\end{abstract}

Keywords: Sys tems, chaos, cli mate, ecol ogy, mod els.

\section{Introducción}

Existe una gran expectativa respecto al calentamiento climático y sus efectos sobre el planeta. La población en general espera que la comunidad científica responda a sus innumerables preguntas y ofrezca una solución viable a los problemas que se presentan en muchos lugares de la Tierra. Las lluvias torrenciales, las inundaciones, las olas de calor y los períodos de sequía, por mencionar algunos ejemplos, inciden de manera directa en el rendimiento de las cosechas, los volúmenes pesqueros y la salud humana, generando efectos importantes en las economías locales y regionales de los sitios donde se producen (Bakun, 1996; Chambers, 1993). Entre los investigadores de todo el mundo especializados en problemas climáticos y ecológicos, prevalecen grandes incertidumbres sobre la mayoría de los temas que atañen a sus áreas de estudio y, a pesar de que se ha buscado y logrado una importante coordinación interdisciplinaria en esta comunidad, los resultados obtenidos hasta ahora no poseen la contundencia que de ellos se espera. ¿A que se debe esto? 
El clima, la ecología y el caos desde la perspectiva de la teoría general de sistemas

Las causas se encuentran en la manera lineal en que se abordan problemas multidimensionales, cuya complejidad desborda por completo los métodos de la ciencia clásica determinista. Ejemplos claros de sus limitaciones, son las dificultades que enfrenta este enfoque cuando trata de elaborar pronósticos sobre la evolución de sistemas complejos, como el clima, la ecología o las sociedades humanas.

Con el fin de apuntalar la necesidad del cambio de paradigma científico que trascienda las restricciones deterministas, en este trabajo se analizarán de forma breve, los elementos que han conducido hacia la visión holística, con la cual, paulatinamente se han encontrado las respuestas que el mecanicismo newtoniano no había podido proporcionar. Estos elementos van, desde la indivisibilidad de lo simple y lo complejo, del todo y sus partes, y del orden y el desorden, hasta la teoría del caos y la teoría general de sistemas.

\section{Hacia la teoría del caos}

La ciencia ha seguido dos caminos a través de su historia; por un lado, la visión mecanicista del mundo, desarrollada principalmente por Galileo, Descartes, Bacon y Newton, entre otros, la cual se basó en la división fundamental de dos reinos separados e independientes entre sí: el espíritu y la materia. El universo material, donde se incluía el organismo humano, era una máquina que, en principio, podía entenderse con sólo analizar por separado sus partes más pe- queñas (Capra, 1991; Ritter et al., 2002). El segundo camino comenzó con Heráclito en el siglo VII a C., quien planteó que el universo presenta armonía en la desarmonía y viceversa. Esta otra visión del conocimiento, vislumbraba lo que después dio pie a la complejidad de la relación orden-desorden-organización, la cual surgió cuando se constató empírica- mente que los fenómenos desordenados del universo son necesarios en ciertas condiciones (Bouald and Marcel, 1988; Balandier, 1989).

Con un ligero vistazo a nuestro alrededor, se advierte una tendencia general al desorden: un ciclón provoca lluvias torrenciales, un tornado genera corrientes de viento intempestivas, una ola de calor desencadena muerte... nunca al revés; pero, contrariamente a lo que se piensa, este desorden no implica confusión. Así entonces, surgió la búsqueda de una explicación de los fenómenos naturales, cuya complejidad los volvía prácticamente irresolubles mediante la aplicación de algoritmos lineales; esto dio pie a un nuevo paradigma: la teoría del caos. El caos es al mismo tiempo muerte-nacimiento, destrucción-creación y tiene que ver con aquello que no podemos saber con certeza; representa a la naturaleza en su creatividad expresada en un vasto campo de conductas y está vinculado con el modo en que ésta crea nuevas formas y estructuras (caos autoorganizado), con su impredictibilidad y su lógica difusa. Los sistemas caóticos se caracterizan por su adaptación al cambio, y en consecuencia, por su estabilidad (Thom, 1972). Si tiramos una piedra a un río, su cauce no se ve afectado, ya que la corriente buscará nuevos caminos; no sucedería lo mismo si el río fuera un sistema ordenado en el que cada molécula de agua tuviera una trayectoria fija.

Las leyes del caos ofrecen una explicación para la mayoría de los fenómenos naturales -desde el origen del Universo, la propagación de un incendio o la evolución de una sociedad- a los cuales es prácticamente imposible predeterminarles un comportamiento con una certidumbre absoluta. Aquí surge la pregunta, ¿por qué lleva la humanidad tantos siglos sumida en el engaño del orden? El problema parte del concepto clásico de ciencia, que exige la capacidad 


\section{N. Sánchez-Santillán y R. Garduño-López}

para pronosticar, de forma precisa, la evolución de un objeto de estudio dado (Chalmers, 1989). Descartes aseguraba que si se fabricara una máquina tan potente que conociera la posición de todas las partículas y utilizara las leyes de Newton para conocer su evolución futura, se podría predecir cualquier cosa del Universo (Garber, 1978). Esta afirmación ilustra la práctica de la ciencia mecanicista a lo largo de su historia; sin embargo, este hito científico que impuso el orden, el determinismo y la predicción en la investigación, acotando igualmente la enseñanza y la divulgación científica, también limitó los estudios a aquellos fenómenos que coincidieran con un patrón previo. Todo lo que evidenciara turbulencia, irregularidad o variabilidad, quedó relegado a la categoría de ruido y cuando éste abarcaba la mayoría de lo observable, los científicos de todas las disciplinas intentaron descomponer a los sistemas en sus partes para tratar de corregir y cuadrar los elementos azarosos y no se afectara el resultado global; no obstante, nada estaba más lejos de la realidad (Koestler and Smythies, 1970).

La condición de cualquier forma de vida es la incertidumbre y la contingencia; la teoría del caos nos invita a cuestionar nuestras creencias y a formular nuevas preguntas acerca de la realidad. Los principales postulados de la teoría del caos son:

- El caos se encuentra en todas partes y se observa un orden dentro de él.

- En la dinámica de los sistemas caóticos, existe una dependencia crítica de las condiciones iniciales en que se producen.

- No existen fronteras disciplinarias que delimiten el estudio y aplicación de la teoría del caos.

\section{Teoría del caos}

En el desarrollo de la teoría del caos, se sucedieron tres pasos fundamentales. El primero de ellos ocurrió hacia finales del siglo XIX, cuando el físico-matemático Henri Poincaré introdujo el concepto de no linealidad, que contradecía el ideal clásico de los sistemas lineales en los que causa y efecto se identifican plenamente, y la resultante de un fenómeno es igual a la suma de sus partes. La no linealidad implica divergencia entre resultado y origen, y la simple adición de las partes de un hecho no corresponde al producto final, por lo que los algoritmos lineales poco pueden hacer para explicar su dinámica. El problema no era menor, si se considera que un $90 \%$ de los procesos de la naturaleza son de carácter no lineal.

El meteorólogo Edward Lorenz, sin proponérselo, dio en 1960 el segundo paso en el avance de la teoría del caos. Al estar realizando un día simulaciones a partir del comportamiento de los parámetros meteorológicos elementales, copió por error los números de la impresión anterior de sus registros y los introdujo en la computadora; observó que el resultado de las condiciones atmosféricas, a escaso tiempo del punto de partida, divergía parcialmente del obtenido con anterioridad, pero al cabo de pocos meses -ficticios, puesto que eran simulaciones- las pautas perdían la semejanza por completo. Lorenz descubrió que el problema se hallaba en los decimales: el programa guardaba seis, pero para ahorrar espacio él sólo había introducido tres, convencido de que el resultado apenas se resentiría (Lorenz, 1963).

Este incidente aparentemente inocuo, marcó el fin de los pronósticos a largo plazo; Leith (1974) y Lorenz (1993) establecen que la condición inicial se vuelve crítica en pronósticos meteorológicos que van más allá de dos semanas. Este descubrimiento puso de 
El clima, la ecología y el caos desde la perspectiva de la teoría general de sistemas

manifiesto la extremada sensibilidad de los sistemas no lineales, la cual se ilustra con el concepto denominado efecto mariposa o "dependencia crítica de las condiciones iniciales", donde se resalta la influencia que ejerce la más mínima perturbación en el estado en que se origina el sistema sobre el estado final del mismo. El escritor James Gleick (1987) parafrasea: "si una mariposa agita hoy con su aleteo el aire de Pekin, puede modificar los sistemas climáticos de Nueva York el mes que viene". Se establece entonces que cualquier variación, ya sea de una milésima o de una millonésima en alguna variable, forma una pequeña muesca que modificará el sistema hasta el punto de hacerlo imprevisible.

Finalmente, el tercer paso se lo imprimió el carácter no lineal e iterativo de los sistemas en la naturaleza, que con instrucciones muy sencillas origina estructuras sumamente complejas. La mayor parte de la materia va de la abundancia de lo sencillo a la escasez de lo complejo, con una distribución piramidal (Ashby, 1972). Aquí, la palabra complejidad indica la cantidad de elementos de un sistema (estructura), sus interacciones potenciales (función) y el número de estados posibles en que se traducen a través de sus relaciones (organización). De esta manera, la complejidad sistémica está en proporción directa con su variedad y variabilidad y es, por lo tanto, una medida comparativa (Forrester, 1971).

Estas características también se observan en los lenguajes escritos, los cuales parten de las letras y pasan luego por las palabras, frases, párrafos, capítulos y libros, con la peculiaridad de que las letras solas no tienen nada que ver con el significado de las palabras, ni tampoco estas últimas aisladas determinan lo que una frase quiere decir, y así sucesivamente. La letra " $\mathrm{h}$ " no está emparentada con el concepto huracán y la palabra "con" puede acompañar igual a una frase de amor o de odio. Esto significa que cuanto más de cerca se mire un problema en la naturaleza, tanto más borrosa se vuelve su solución. Las estructuras más complejas o altas en la pirámide tienen propiedades ajenas y diferentes a las de niveles inferiores, situación que le genera un problema importante a la ciencia, al perder ésta su capacidad de predicción; a dichas propiedades se les conoce como propiedades emergentes (Morin, 1994). Sin embargo, si la precisión difumina aún más el objeto de estudio, ¿qué estrategia debe emplearse para estudiar los sistemas complejos? Aquí interviene la teoría de la totalidad, que concibe el mundo como un todo orgánico, fluido e interconectado; si algo falla no debe buscarse la porción dañada, sino más bien hay que revisar el sistema completo, ya que se trata de una unidad indisoluble (Morin, 1994).

Resulta incompleto tratar de observar la naturaleza de modo fragmentado y explicarlo todo mediante la suma de sus partes, ignorando dos aspectos primordiales: la imposibilidad de "meter la totalidad en una bolsa", porque ésta también forma parte de aquella, y la dependencia que existe entre el observador, lo observado y el proceso de observación: dado que el hombre forma parte de la totalidad, su percepción y su mera presencia altera el objeto de estudio. El caos se debe interpretar desde el punto de vista global para salvar las fronteras de las diferentes disciplinas y aceptar la paradoja que convierte lo simple y lo complejo, el orden y el caos en elementos inseparables, donde la palabra "elementos" define a las partes o componentes de un sistema que pueden organizarse en un modelo. Ejemplo de esta paradoja es el denominado fractal de Mandelbrot (1983), una de las concepciones más complejas que ha imaginado el hombre, el cual se creó a partir de una ecuación iterativa muy simple. Desde esta visión, el 


\section{N. Sánchez-Santillán y R. Garduño-López}

caos se convierte en una inagotable fuente de creatividad de la que también puede surgir el orden, proceso que puede revertirse, generándose entonces caos a partir de la armonía. Las civilizaciones antiguas creían en la armonía entre el caos y el orden, y definían al primero como "una suerte de orden implícito"; quizá sea el momento de reflexionar y retomar el concepto.

\section{Teoría general de sistemas}

La reflexión de las relaciones entre lo simple y lo complejo, el todo y sus partes, el orden y el desorden, conduce directamente a b teoría general de sistemas, paradigma fundamental para abordar un sistema complejo como lo es, por ejemplo, el clima, cuyo estudio era, hasta no hace mucho tiempo, una subdisciplina o subsistema de la Geografía, entendiendo como subsistema, al conjunto de elementos y relaciones que responde a estructuras y funciones especializadas dentro de un sistema mayor. En términos generales, los subsistemas tienen las mismas propiedades que los sistemas y su posición es relativa a la del observador. Desde este punto de vista se puede hablar de subsistemas, sistemas o supersistemas.

Ludwing Von Bertalanffy, fue alrededor de 1930, uno de los pioneros de esta innovadora teoría, seguido por Ronald Ashby y Gregory Bateson en las siguientes dos décadas. Las propuestas maduraron hasta convertirse en la base de la teoría general de sistemas, cuya construcción teórica trata de los principios y leyes que les conciernen a éstos, sin importar la rama científica de la que provengan y donde se busca una formalización matemática entre las relaciones de sus componentes y sus funciones isomorfas. Estas últimas se refieren a las características del mundo material e inmaterial que afectan a los sistemas (Bertalanffy, 1973). Varios elementos que contribuyeron en la elaboración de este nuevo paradigma fueron aportados por la teoría de las organizaciones, la cibernética y la teoría de juegos (Ashby, 1972; 1984).

La teoría general de sistemas surge en contraposición a la corriente mecanicista y determinista (Grun, 1995), cuya visión o paradigma estaba fundamentado en dos principios: 1) que es posible entender completamente el mundo y 2) que dicho entendimiento se podía lograr mediante el método analítico. Ambos principios se basaban en el reduccionismo (Popper, 1983), el cual implica dividir todo hasta sus últimos elementos, a partir de los cuales se explica luego el resto; los átomos en la física o la célula en la biología son ejemplos de esto. No obstante, con los conocimientos actuales en ambos campos se ha encontrado que estos elementos también se pueden dividir. El método analítico consiste en desarmar en partes discretas aquello que se pretende entender, tratar de explicar el comportamiento de las partes separadas, y luego amalgamar el entendimiento de éstas en una comprensión de la totalidad. Un postulado determinista crucial es la consideración de que todos los fenómenos se pueden explicar a través de relaciones causa-efecto (Popper, 1983). El problema surge cuando se intenta explicar un efecto excluyendo varios factores, pasando de lado el hecho de que en todo fenómeno inciden una multiplicidad de causas y no sólo es producto de una de ellas.

Esta línea de pensamiento ha creado problemas prácticamente irresolubles, los más importantes, derivados de lo que se denomina la antinomia del determinismo, donde el libre albedrío y las dificultades de verificar leyes sobre la base de la causalidad implican crear condiciones ideales (Popper, 1983). Uno de los ejemplos clásicos es el estudio de la gravedad a través de la simulación de la caída de los cuerpos en el vacío 
El clima, la ecología y el caos desde la perspectiva de la teoría general de sistemas

absoluto, dentro del laboratorio. Otros dos ejemplos comunes en el campo de la meteorología, son el estudio de los tornados y los huracanes, en los que el enfoque principal se da al comportamiento de la presión barométrica y la temperatura, dejando de lado la circulación zonal, las celdas anticiclónicas y las corrientes marinas. Hacia finales de la segunda guerra mundial, parte de la comunidad científica reconoció que algo andaba mal. Comenzó a verse que al analizar un sistema, sus propiedades esenciales se perdían; por ejemplo, un automóvil desarmado no transportaba a una persona "desarmada"; advirtieron que era necesario un pensamiento holístico, (del griego holos = total), es decir, que abordara los problemas como un todo para explicar las cosas. Así, se buscaba revelar cómo es que el sistema está estructurado, por qué funciona de cierta manera y para qué lo hace. Es importante señalar que ésta concepción abarcó al método analítico, por lo cual análisis y síntesis son complementarios, y el pensamiento sistémico incluye a ambos.

Los principales objetivos de la teoría general de sistemas son:

- Investigar las analogías, paralelismos, semejanzas, correlaciones e isomorfías de los conceptos, leyes y modelos en las diversas ciencias. Isomorfía se define como la fórmula, pauta, estructura, proceso o interacción que demuestra ser la misma, sólo en términos generales, en diversas disciplinas y escalas de magnitudes dentro de los sistemas reales y cambia en función del sistema que se analice.

- Fomentar la transferencia de conocimientos entre las diversas ciencias.

- Estimular el desarrollo y formulación de modelos teóricos en aquellos campos que carecen de ellos, o en los cuales los mismos son rudimentarios e imperfectos.

- Promover la unidad de las ciencias y tratar de obtener la uniformidad del lenguaje científico.

De manera paralela, la cibernética se ocupa del estudio del mando, el control, las regulaciones y el gobierno de los sistemas, por lo que constituye una parte inseparable de la teoría general de sistemas; sus conceptos resultan extremadamente útiles para entender el funcionamiento de los sistemas complejos como el clima, los ecosistemas o el derecho, por mencionar algunos. Dentro de este paradigma, el sistema es una entidad autónoma dotada de una cierta permanencia, que está constituida por elementos que se conforman como subsistemas, estructural y funcionalmente interrelacionados, y cuya transformación ocurre dentro de ciertos límites de estabilidad, gracias a autorregulaciones que le permiten adaptarse a las variaciones de un entorno específico (Thom, 1972). Así, la teoría general de sistemas, estudia a éstos con todas sus conexiones internas (estructura primaria), y externas (hipoestructura) (Lugo y Morris, 1982). Sus elementos no se pueden separar sin alterar la esencia del sistema, es decir, su unidad, pues una de las ideas básicas de esta teoría es que el todo es más que la suma de sus partes, porque las características del todo no son explicables a partir de las características de las partes aisladas. La entidad de nivel superior tiene otras propiedades o atributos diferentes a las de los niveles inferiores (Peters, 1985).

Uno de los requerimientos de todo sistema es el de constituir una entidad aislada, aunque su aislamiento no es absoluto, pero sí lo suficiente para poder distinguirlo de su entorno, clara y permanentemente. De acuerdo con Grun (1995), un sistema biológico está 


\section{N. Sánchez-Santillán y R. Garduño-López}

rodeado por un límite o membrana que lo aísla relativamente y separa el endomundo del exomundo. Las membranas son siempre permeables y selectivas, y juegan un papel fundamental en la organización de los intercambios entre el sistema y su entorno (o, eventualmente, en los que operan entre subsistemas, limitados ellos mismos, por membranas). Los sistemas metavivientes también desarrollan membranas en sentido amplio: las fronteras políticas, los recintos de las empresas y organizaciones -en el caso de las sociedades humanas- o los límites de las termiteras -para las sociedades animalesconstituyen membranas en el sentido antes definido, es decir, son funcionalmente homólogas a las membranas biológicas (Rodríguez y Arnold, 1991).

Margalef (1980), señala que un sistema es algo factible de ser disecado, o sea, que se pueden reconocer partes separables actuando unas sobre otras; cambia con el transcurso del tiempo, pero conserva algunas propiedades invariantes y casi todo lo existente puede definirse con este concepto. Un sistema se concibe entonces conformado por elementos (estructura), e interacciones (función), que vinculan a estos componentes. Las relaciones pueden ser recíprocas (principio de circularidad) o unidireccionales. El resultado de estas interacciones es la organización del sistema, que cuando persiste, genera posibles escenarios que no son completamente indeterminados o regidos por el azar. Es factible por lo tanto, aportar una descripción suficiente de un sistema completo en una forma abreviada o corta. Un aspecto fundamental de los sistemas es la existencia de restricciones, que limitan el ámbito dentro del que se sitúan sus estados futuros (Bohm and Peat, 1987).

Cuando un elemento o un subsistema se combina con otros en el interior de un sistema, aparece cierta convergencia de comportamiento, en el sentido de que cada elemento influye sobre las posibles variaciones de los demás y, como consecuencia, el número de posibilidades que a priori podría parecer muy grande, queda más o menos limitado (Peters, 1985). Un sistema tiende a converger o a cerrarse sobre sí mismo; en caso contrario diverge, con posibilidades de extinguirse. Puesto que cada acción depende de la conformación de las influencias precedentes, las posibilidades de variación se reducen y la actividad del sistema parece estar guiada o regulada. En un sentido amplio, se comporta en forma cibernética en su regulación y control (Ashby, 1972). Un sistema se caracteriza por la convergencia en el comportamiento de sus diversas partes. En consecuencia, el conjunto de los estados del sistema es menor que el de los posibles, emanados de no conocer las interacciones entre las partes. La propiedad básica de todo sistema consiste en la restricción del número de estados o configuraciones realizables, por lo tanto, todo sistema contiene información, con el significado que la teoría de la comunicación le da a esta palabra (Ashby, 1984).

\section{Memoria de los sistemas y principio de asimilación del más débil}

Las fronteras e interfases son muy importantes por su capacidad de acumular información, la cual se refleja en la riqueza de límites reconocibles; constituyen los contornos que separan a los sistemas y en todos los casos es el observador quien define lo que pertenece y lo que queda fuera de ellos (Margalef, 1980). La información de los sistemas siempre está asociada a la historia de su desarrollo y en un mundo que ya posea cierta estructura, cualquier interacción entre la materia y la energía modifica esta disposición y hace a los cambios futuros más predecibles con respecto a su momento anterior. La cantidad de información que 
El clima, la ecología y el caos desde la perspectiva de la teoría general de sistemas

contiene el sistema es igual a la que ya existe, más la que entra, produciéndose una agregación neta en esta entrada, ya que la salida de energía no elimina información de él (Johansen, 1975). Toda la energía que se incorpora a los sistemas obedece la ley de la conservación de ella, es decir, la cantidad que permanece en un sistema es igual a la suma de la energía importada -de un nivel a otromenos la suma de la energía exportada, denominada negentropía (Ashby, 1984).

Asimismo, Margalef (1980) señala que la información que está presente en las estructuras actuales sirve para reconstruir el pasado y refleja verazmente la energía usada y degradada con anterioridad. Esta energía no se ha perdido por completo, puesto que las estructuras que se formaron guardan la información para canalizar cambios futuros $\mathrm{o}$, dicho de otra manera, hace que determinados estados futuros sean más probables que otros. Es posible descubrir o interpretar la utilidad de dicha información, dado que las estructuras acumuladas hacen más eficiente la degradación de la energía.

Así, la acumulación de información significa cambios de energía y, por lo tanto, un aumento de la entropía, parámetro que mide el grado de desorden del sistema (Cesarman, 1984). Sin embargo, la información conseguida persiste en forma de estructura $y$ puede orientar en uno $\mathrm{u}$ otro sentido el uso futuro de la energía, de una manera que se puede juzgar como más eficiente; esto implica que cuando la información sube de categoría o de estrato se adquiere una mayor cantidad de ella, que se traduce en otras posibilidades de organización de canales y códigos, y la información resultante que ingresa al sistema adquiere un nuevo sentido. Al proceso de introducción de nuevos resultados al sistema en forma de retroalimentación se le denomina recursividad (Bertalanffy, 1973). Si se aplicara lo anterior al contexto de las sociedades humanas, significaría que la acumulación de información que realizan las sociedades tecnológicamente más avanzadas es mayor a las de menor avance; la misma situación se da en la naturaleza entre los ecosistemas, de acuerdo a su grado de desarrollo.

Este efecto resulta particularmente importante en el estudio de intercambios entre sistemas. El sistema que es mayor, en términos de organización adquirida, puede hacer siempre un mejor uso de la información, es decir, la asimila y la multiplica. Según Margalef (1957), tal asimetría en el intercambio de información se percibe como injusta, puesto que el participante con más información, recibe aún más; esta constatación se le designa como el principio de San Marcos, por la relación que guarda con el evangelio según San Marcos (versículo 4:25), que dice: al que tiene se le dará más y al que no tiene, aún lo poco que tiene se le quitará. En conclusión, cuando dos sistemas con diferente complejidad interactúan, el aumento de la información es mayor en el más complejo, pues parece alimentarse del más simple e, incluso, puede asimilarlo.

\section{Estructura más funcionamiento igual a organización. La fuerza estabilizadora}

En el terreno de la ecología o de la climatología, analizadas desde la teoría general de sistemas, el principio de San Marcos resulta extraordinariamente valioso al aumentar todos los gradientes de las variables portadoras de información, propiedad que puede contribuir a la división progresiva del espacio.

Dichos efectos son particularmente importantes en la creación de bloques discontinuos que funcionan como piezas en los sistemas abiertos y se encuentran lejos de una 


\section{N. Sánchez-Santillán y R. Garduño-López}

situación de equilibrio. Lo anterior ejemplifica cómo la estructura puede crecer alimentándose de la función. Sin embargo, los estados de equilibrio sistémico, denominados equifinalidad y multifinalidad, pueden alcanzarse en los sistemas abiertos por una diversidad de caminos; la permanencia de dichos estados implica, de manera necesaria, la importación de recursos provenientes del ambiente. Los recursos pueden consistir en flujos energéticos, materiales o informativos (Asbhy, 1984).

La realidad se presenta bajo dos aspectos complementarios e inseparables:

\section{1) lo estructural-estático y}

2) lo funcional-dinámico, en donde cada elemento se halla situado en la estructura de acuerdo con la función que le compete (Ashby, 1984). Estructura y función son dos formas complementarias de una misma realidad y ninguna describe, aisladamente $\mathrm{y}$ en forma total, al sistema objeto de estudio.

Sin estructura, la función desaparecería. En un sistema con enfoque diacrónico se resalta la función, mientras que en uno sincrónico, la estructura; no obstante, un modelo estructural-funcional reconoce que los dos aspectos deben integrarse adecuadamente.

Así, ningún modelo sistémico puede ser estático, porque ningún sistema lo es, salvo quizás en el instante en que deja de ser un sistema y empieza a descomponerse en sus elementos (Margalef, 1980). En todos los casos, los modelos son construcciones diseñadas por un observador que persigue identificar y mensurar las relaciones sistémicas complejas; asimismo, todo sistema real puede ser representado por más de un modelo, cuya expresión depende tanto de los objetivos del modelador, como de su capacidad para distinguir las relaciones relevantes enfocadas a tales objetivos; de cualquier manera, la esencia de los modelos sistémicos es la simplificación (Ísita, 2002).

De la cibernética proviene el concepto de retroalimentación, el cual implica que todos los elementos, componentes o subsistemas de un sistema se comunican entre sí para desarrollar interrelaciones coherentes, es decir, que favorezcan la permanencia del sistema en el tiempo y en el espacio (Margalef, 1980). La retroalimentación es negativa cuando su función consiste en contener o amortiguar el cambio y se le denomina fuerza estabilizadora, aunque no siempre es una fuerza en el sentido físico de la palabra. La retroalimentación es positiva si amplifica o multiplica el cambio en una dirección determinada (Ashby, 1984).

Existe una conexión de la información y su forma específica de procesarla en la meteorología, la climatología, la ecología, la biología social e incluso, en el derecho, donde no es admisible proponer jerarquías científicas sino, más bien, una interdependencia entre cada una de las ciencias mencionadas para dar paso a una visión sistémica y holística que transformaría la perspectiva analítica, determinista y reduccionista por el simple hecho de incluir la retroalimentación como parte del proceso. Este es el enfoque más adecuado para estudiar el calentamiento climático global, dado que el análisis sistémico permite visualizar de manera integral los aspectos sociales, económicos, políticos, jurídicos, ecológicos y climáticos, al contemplar la realidad desde distintos puntos de vista y poder trabajar inter y transdiciplinariamente (Lovelocket al., 1992).

El analizar los problemas bajo la óptica de los sistemas complejos, sean humanos, sociales, físicos o ecológicos, implica elaborar o modificar sus formas con el objeto de conservar viable el sistema a través de 
El clima, la ecología y el caos desde la perspectiva de la teoría general de sistemas

retroalimentaciones positivas o negativas. En estas condiciones, los procesos apuntan al desarrollo, la estructura, al crecimiento o cambio de forma, así como al estado general del sistema. En términos cibernéticos, los procesos causales mutuos que se refieren a la circularidad o retroalimentación, se denominan morfogenéticos. Estos procesos activan y potencian la posibilidad de adaptación de los sistemas a ambientes en transición.

Por otro lado, la morfostasis se refiere a los procesos de intercambio que se generan con el ambiente y tienden a mantener un estado ya existente con una retroalimentación negativa; un ejemplo de éstos, son los sistemas vivos (Lovelock and Margulis, 1973). Lovelock et al. (1992), proponen un sistema jurídico global que abarque el sistema ecológico del planeta y dan a este concepto el nombre de Gaia. Consideran al hombre y a los ecosistemas como un solo organismo viviente, por lo que resulta imprescindible diseñar una legislación, establecer una jurisprudencia y elaborar una doctrina que considere esta unidad (Lovelock and Margulis, 1973). Con esta visión, derecho y ecología se retroalimentan a través de redes complejas, de una forma quizá más bien caótica, pero mucho más creativa de lo que parece a simple vista, siempre bajo los límites flexibles sugeridos por la termodinámica y la teoría de juegos.

\section{Sistemas y modelos}

Los sistemas pueden agruparse en reales, ideales y modelos. Los primeros tienen una existencia independiente del observador, quien los puede descubrir; los segundos son construcciones simbólicas; al tercer tipo corresponden las abstracciones de la realidad que combinan lo conceptual con las características de los objetos.

Un modelo es cualquier abstracción o simplificación de un sistema, cuyo propósito es la conceptualización, organización y comunicación de un fenómeno. Si bien los modelos simplifican los fenómenos reales, conservan los atributos funcionales del sistema (Hall and Day, 1977).

Los tres pasos básicos para la elaboración de un modelo determinista son: crear un mapa conceptual, trazar un esquema diagramático que lo represente y desarrollar su expresión matemática. En el mapa conceptual se utilizan los paradigmas científicos vigentes, se toman en cuenta los componentes, las interacciones y los mecanismos que creemos relevantes para que opere el sistema y, de manera particular, el fenómeno que se quiere estudiar. El esquema diagramático se hace a través de la construcción de com- partimentos o cajas que representan las variables de estado del sistema, y de flechas, que señalan los flujos de materia y energía (Shoemaker, 1977).

Las expresiones matemáticas pueden incluir relaciones lineales o no lineales, discretas o continuas, y determinísticas o estocásticas. La mayoría de los modelos se conceptualizan por medio de ecuaciones diferenciales. En el caso de los modelos lineales, la elección entre las variables es arbitraria, porque uno puede trasladar la materia o energía de una variable a otra; en el caso de los no lineales, las traslaciones no son unidireccionales. Las ecuaciones de los modelos determinísticos incorporan tasas de cambio en el tiempo, lo que permite realizar predicciones (Shannon and Weaver, 1969); sin embargo, si se cambian las condiciones iniciales, aunque sea por una cantidad ínfima, tenderá a modificarse la predicción, ya que el error se amplifica en el tiempo a un ritmo exponencial (Lorenz, 1963). 


\section{N. Sánchez-Santillán y R. Garduño-López}

\section{La creatividad y la teoría general de sistemas}

El mundo confuso y desordenado, con estilos de vida prosaicos y estériles, así como empleos que a veces inspiran poco, nos conduce al caos; sin embargo, esto se puede traducir en grandes oportunidades, pues la visión sistémica nos da la posibilidad de proyectar y adaptar un futuro nuevo. La creatividad con enfoque sistémico es un estilo de vida que incide en el pensamiento, la conducta, las actitudes, los valores, la comunicación, la investigación, la enseñanza y la divulgación, entre otros aspectos. Consiste en ver las cosas con un nuevo enfoque y hace del proceso de aprendizaje algo permanente; en otras palabras, nos convierte en aprendices vitalicios. Involucra transformaciones en las que las verdades eternas dejan de ser inamovibles, a menos que tengan significado para cada situación innovadora; en resumen, se convierte en un medio para lograr algo mejor y más maduro. Esta creatividad tiene como característica principal la de ser permanentemente constructiva, al fundir pensamientos y acciones en una configuración novedosa y relevante; permite percibir los problemas, deficiencias y faltas de armonía y encuentra relaciones nuevas con la información existente. Se convierte en una herramienta que identifica los elementos ausentes para encontrar soluciones, partiendo de hipótesis perfectibles. Genera pensamientos y acciones innovadoras que están en función del conocimiento, la imaginación y la evaluación -donde el proceso es tan importante como el producto resultante- a la vez que facilita comunicar resultados eficientemente. En el hombre moderno, la mente finita no alcanza a comprender la complejidad y la verdad infinitas; le gustan las ideas o fórmulas sencillas, congruentes y uniformes; le desagradan la tensión, la ambigüedad y la oposición; en fin, es un ser agobiado, un tanto marchito por las preocupaciones que oscurecen todo lo que le rodea.

Si los esfuerzos de investigación, enseñanza y divulgación científicas se fortalecen con ideas creativas, se conjuran, en gran medida, las empresas estériles y condenadas al fracaso. Ser del mismo sentir, no significa necesariamente tener la misma opinión, pues el objetivo es desarrollar, deliberada y conscientemente la conducta creativa y el potencial humano, además de identificar lo que no se puede cambiar y lo que sí se debería, permitiendo abrir el horizonte hacia alternativas innovadoras. La capacidad de deducción, a partir de las leyes de la lógica y las matemáticas, crea pensadores disciplinados, aunque no forzosamente mentes imaginativas; de ahí que las personas más inteligentes, no son necesariamente las de mayor inventiva. Algunas sociedades promueven la creatividad, mientras que otras la inhiben. La educación es un proceso sin fin y aquél que deja de aprender no sólo se marchita, sino que ahoga a quienes lo rodean. A medida que la sociedad experimenta cambios profundos, rápidos y significativos, los métodos de enseñanza y divulgación también deben ser diferentes; una persona creativa debe abrazar lo nuevo e inexplorado por ser ésta una oportunidad de crecimiento (Ritter y Klimek, 1997) y se le debiera alentar en la difusión de sus propuestas.

\section{Referencias}

Ashby W.R. (1972). Introducción a la cibernética. Edito rial Nueva Visión, Buenos Aires.

Ashby W.R. (1984). Sistemas y sus medidas de información. En: Tendencias en la teoría General de Sistemas (Bertalanffy L. Von, Ashby R.W., Weinberg G.M.), 3a ed, Ed. Alianza, Madrid. 
El clima, la ecología y el caos desde la perspectiva de la teoría general de sistemas

Bakun A. (1996). Patterns in the Ocean: Ocean Processes and Marine Population Dynamics. Ed. California Sea Grant College System, National Oceanic and Atmospheric Administration, en cooperación con el Centro de Investigaciones Biológicas del Noroeste, La Paz, Baja Cali fornia Sur.

Balandier G. (1989). El desorden. La teoría del caos y las ciencias sociales. Elogio de la fecundidad del movimiento. Ed. Gedisa, Barcelona.

Bertalanffy L.V. (1973). General System Theory: Foun dations, Devel op ment, Applications. Alan Lane. Ed. George Braziller, New York.

Bohm D. and Peat D. (1987). Science, Order and Creativity. Ed. Bantam Books, Toronto.

Bouald-Ayoub J. and Marcel H. (1988). Culture, Signes, Critique. Séries: Cahiers Recherches et ThéoriesCollection Symbolique et Idéologie; S16. Montreal: Dep. de Philosophie (UQAM).

Capra F. (1991). El nuevo paradigma ecológico. Ed. Nueva Conciencia. Integral, Barcelona.

Cesarman E. (1984). Hombre y entropía. Ed. Pax-México, México.

Chalmers A.F. (1989). ¿Qué es esa cosa llamada ciencia?: Una valoración de la naturaleza y el estatuto de la ciencia y sus métodos. $8^{\mathrm{v}}$ ed. Ed. Siglo XXI, México.

Cham bers F.M. (1993). Climate Change and Human Impact on the Landscape: Studies in Palaecology and Environmental Archaeology. Ed. Chapman and Hall, London.

Forrester J.W. (1971). World Dynamics. Ed. Wright-Allen Press. Cambridge Mass.

Garber D. (1978). Science and Certainly. In: Descartes: Critical and interpretative essays (Hooker M), Ed. Johns Hopkins Univer sity Press, Balti more.
Gleick J. (1987). Chaos: Making a New Science. Ed. Penguin Books, New York.

Grun E. (1995). Una visión sistémica y cibernética del derecho. Ed. Albedo Perrot, Argen tina.

Hall A.S. and Day W.J. (1977). System and Models: Terms and Basic Principles. In: Ecosystem Modeling in Theory and Practice: An Introduction with Case Histories (Hall A.S. and Day W.J.), Ed. John Wiley and Sons, New York.

Ísita R. (2002). Divulgación persuasiva de la ciencia. En: Antología de la divulgación de la ciencia en México (Tonda J., Sánchez A., Chávez N.), colección: Divulgación para Divulgadores. Dirección General de Divulgación de la Ciencia, UNAM, México.

Johansen B.O. (1975). Las comunicaciones y la conducta de la organización. Ed. Diana, México.

Koestler A. and Smythies J.R. (1970). Das neue menschenbild: Die Revolutionierung der Wissenschaften Vom Leben, Ein Intenationales Symposion. Verlag Fritz Molden, München.

Leith C.E. (1974). Theoretical Skill of Monte Carlo Fore casts. Monthly Weather Review, 102, pp. 409-418.

Lorenz E.N. (1963). Deterministic Nonperiodic Flow.Journal of the Atmospheric Science, 20, pp. 130-141.

Lorenz E.N. (1993). The Essence of Chaos. University of Washington Press, Seattle.

Lovelock J.E. and Margulis L. (1973). Atmospheric Homeostasis by and for the Biosphere: The Gaia Hypothesis. Tellus, 26, pp. 2-23.

Love lock J.E., Clarke J. y Orive B. (1992). Gaia: una ciencia para curar el planeta. Ed. Inte gral. Serie: Los libros de Integral. No. 51, Barce lona.

Lugo A.E. y Morris G.L. (1982). Conceptos del análisis de sistemas. En: Los sistemas ecológicos y la humanidad. 


\section{N. Sánchez-Santillán y R. Garduño-López}

Secretaría General de la OEA. Programa Regional de Desarrollo Científico y Tecnológico. Washington, D.C. EUA. Serie Biología. Monografía, N²3. p. 82.

Mandelbrot B.B. (1983). The Fractal Geometry of Nature. Ed. W.H. Freeman, San Francisco.

Margalef R. (1957). La teoría de la información en ecología. Memorias de la Real Academia de Ciencias y Artes. Tercera Época, Barce lona, 32(3), pp. 373-445.

Margalef R. (1980). La biosfera: entre la termodinámica y el juego. Ed. Omega, Barcelona.

Morin E. (1994). Introducción al pensamiento complejo. Ed. Gedisa, Barce lona.

Peters R.H. (1985). Predic tion and Understanding Alternative Goal in Science. Conferencia ofrecida en el Simposium Reductionism versus Holism. Universidad de Ottawa.

Popper K. (1983). Conjeturas y refutaciones: el desarrollo del conocimiento científico. Ed. Paidós, Barce lona.

Ritter W. y Klimek G.R. (1997). La relación ciencia-educación y la bús- queda de un nuevo perfil académico. Ciencia y Mar, 1(1), pp. 31-35.

Ritter W., Guzmán S., Sánchez-Santillán N., Suárez J., Corona-Muñoz C., Ramos A., Rodríguez R. y Pérez T. (2002). El clima como sistema complejo adaptativo en coevolución. Ciencia y Mar, VI (17), pp. 23-25.

Rodríguez D. y Arnold M. (1991). Sociedad y teoría de sistemas. Ed. Universitaria, Santiago de Chile.

Shannon C.E. and Weaver W. (1969). The MathematicalTheory of Communication. Univer sity of Illi nois Press, Illi nois.

Shoemaker C.A. (1977). Mathematical Construc tion of Ecolog ical Models. In: Ecosystem Modeling in Theory and Practice: An introduction with case histories (Hall A.S., Day W.J.), Ed. John Wiley \& Sons. New York.

Thom R. (1972). Stabilité Structurelle et Morphogenèse: Essai d'une Théorie Générale des Modèles. W.A. Benjamin. Ed. Reading, Massa chu setts.

\section{Semblanza de los autores}

Norma Sánchez-Santillán. Realizó sus estudios en hidrobiología en la Universidad Autónoma Metropolitana, plantel Iztapalapa. Obtuvo el grado de maestra en ciencias (Biología), orientada en aspectos de climatología en la Facultad de Ciencias de la UNAM en 1994. Posteriormente, alcanzó el grado de doctora, siguiendola línea de aplicación climática en aspectos de ecología (1999). Cuenta con un diplomado en divulgación de la ciencia, por parte de la Dirección General de Divulgación de la Ciencia, UNAM (2004). Es autora de 70 trabajos publicados en revistas con arbitraje; así como 55 artículos de divulgación en revistas arbitradas. Pertenece al Sistema Nacional de Investigadores desde el año 2002. Actualmente se desarrolla como investigadora en la UAM-Xochimilco, como responsable del Laboratorio de Climatología, así como profesora dentro del plantel.

René Garduño-López. Obtuvo la licenciatura en física y el posgrado en geofísica por la Facultad de Ciencias de la UNAM, en donde es profesor desde 1976. Es investigador titular del Centro de Ciencias de Atmósfera (CCA) de la UNAM. Ha publicado 24 artículos de investigación en revistas arbitradas y siete capítulos en libros. Es editor asociado de la revista Atmósfera (incluída en Science Cita tion Index). Es autor de los libros El veleidoso clima y Pormenores terrestres, del Fondo de Cultura Económica. En el CCA ha sido secretario académico y actualmente es jefe de departamento. 